Published in final edited form as:

Mol Psychiatry. 2020 September ; 25(9): 2189-2199. doi:10.1038/s41380-018-0259-2.

\title{
Socioeconomic status and inflammation: a meta-analysis
}

\author{
Keely A. Muscatell ${ }^{1,2}$, Samantha N. Brosso ${ }^{1}$, Kathryn L. Humphreys ${ }^{3}$ \\ ${ }^{1}$ Department of Psychology \& Neuroscience, University of North Carolina at Chapel Hill, Chapel \\ Hill, NC, USA \\ ${ }^{2}$ Lineberger Comprehensive Cancer Center, University of North Carolina at Chapel Hill, Chapel \\ Hill, NC, USA \\ ${ }^{3}$ Department of Psychology and Human Development, Vanderbilt University, Nashville, TN, USA
}

\section{Abstract}

Socioeconomic status (SES), often conceptualized as income, education, or occupation, is associated with risk for disease morbidity and psychopathology. Recent research has focused on the potential biological mechanisms linking lower SES and poor outcomes; much of this work has examined the relationship between SES and markers of systemic inflammation. The strength of the estimated association between SES and inflammatory markers varies widely across individual studies. Thus, we used meta-analytic techniques to quantify the magnitude of this relationship. To accomplish this, PubMed and PsycINFO were searched for papers that reported on SES and two commonly measured systemic inflammatory markers, C-reactive protein (CRP) and interleukin-6 (IL-6). Peer-reviewed, empirical papers conducted in non-patient populations were included. Data from 43 papers $(N=111,156)$ reporting a total of 63 relevant effect sizes were included in analyses. SES, broadly defined, was significantly associated with both levels of CRP ( $Z=0.12$; 95\% CI, 0.09-0.16) and IL-6 ( $Z=0.15$; 95\% CI,0.12-0.18); individuals with lower SES showed higher levels of systemic inflammation. Subanalyses demonstrated that studies operationalizing SES as either levels of income or educational attainment also found significant associations with both CRP and IL-6. Moderator analyses revealed that effect sizes varied based on sample characteristics and analysis approaches. Lower SES is associated with significantly elevated levels of inflammatory markers of disease risk. Thus, proinflammatory pathways are likely an important mechanism translating socioeconomic inequalities into mental and physical health disparities.

\section{Introduction}

Socioeconomic gradients in numerous negative physical and mental health outcomes are well-established [1,2]. Indeed, factors such as poverty, low educational attainment, and neighborhood deprivation are associated with increased risk for psychopathology, including

Keely A. Muscatell kmuscatell@unc.edu.

Conflict of interest Dr. Humphreys received financial support from NIH, the Brain and Behavior Research Foundation, the Klingenstein Third Generation Foundation, and the Jacobs Foundation. Dr. Muscatell and Ms. Brosso declare that they have no conflict of interest.

Electronic supplementary material The online version of this article (https://doi.org/10.1038/s41380-018-0259-2) contains supplementary material, which is available to authorized users. 
major depression [3] and schizophrenia [4], as well as poor physical health outcomes, including cardiovascular disease [5], diabetes [6], and all-cause mortality [7]. More recently, research has begun to investigate the biological mechanisms by which lower socioeconomic status (SES) confers risk for negative outcomes. Increases in systemic inflammation have emerged as one possible physiologic pathway. Levels of the inflammatory markers Creactive protein (CRP) and interleukin-6 (IL-6) have been shown to prospectively predict a number of negative outcomes in pre-clinical and clinical studies, including depressive symptoms [8], likelihood of coronary events [9], ischemic stroke incidence [10], and allcause mortality [11]. Further, inflammatory processes are upregulated in response to psychological stress [12], obesity [13], and among cigarette smokers [14], all of which are more prevalent in lower SES populations. Thus, increased levels of systemic inflammation may be a critical link between SES and health outcomes.

Associations between SES and inflammation were first reported nearly two decades ago [15-18]. Since then, numerous studies have examined associations between various indices of SES and levels of inflammatory markers. Estimates of the association between SES and inflammation vary widely across studies, likely due in part to the use of different measures of SES, different markers of inflammation, demographic characteristics of the samples, and other methodological factors. As such, the strength of the relationship between SES and inflammation is difficult to ascertain based on any single study from the current literature. Thus, the purpose of the present study was to use meta-analytic techniques to quantify the association between SES and the two most commonly assessed markers of systemic inflammation (i.e., CRP, IL-6). SES was broadly defined, including measures of objective (e.g., income, education, occupation), subjective (i.e., perceived social status), and neighborhood (e.g., average neighborhood income) SES. We also conducted analyses focusing exclusively on income and education, respectively. Finally, we explored potential moderators of SES -inflammation associations, focusing on sample characteristics and study design choices, to inform future research in this area.

\section{Methods}

\section{Search strategy and selection criteria}

We employed two primary search strategies (see Fig. 1 for PRISMA flowchart) to identify the 43 studies included. First, we conducted searches through PubMed and PsycINFO in April 2017 for studies that examined both SES and either CRP or IL-6 (see Supplementary Material for full search terms). Second, we reviewed the references of identified papers for possible additional studies, using forward and backward searching. Authors of journal articles that met the inclusion criteria but did not include the data required to calculate an effect size were contacted for additional information $(N=11)$.

Each study was required to satisfy the following criteria to be included in the overall metaanalysis: (a) assessed SES; (b) assessed circulating levels of CRP and/or IL-6; (c) provided data to determine a relevant effect size; and (d) included only North American participants. We restricted the analysis to studies conducted with North American samples in an effort to account for the fact that socioeconomic influences on health vary strongly across countries and continents [19]. Studies were excluded if they focused on participants with an existing 
chronic condition or disease (e.g., diabetes) in order to eliminate studies that could bias the estimates, given that patients may be substantially non-representative in terms of their levels of inflammation.

\section{Data extraction}

Two trained raters (KAM, SNB) coded individual studies. When more than one relevant effect size was available, raters first selected the effect size with the fewest covariates included in the model, and second, the variable with the strongest association with the inflammatory marker of interest, as often the models included several variables that competed for explanatory variance (see Supplementary Information for more detail). Rater agreement for moderator codes was $96 \%$. When raters provided contradictory judgments, disagreements were resolved via consensus.

\section{Data analysis}

To ensure consistency in the directionality of the effect sizes, SES was coded to indicate that numerically higher SES values indicated lower SES. Effect size calculations were obtained using standard approaches, given each association available (e.g., r, two continuous variables; $d$, one continuous and one binary variable; odds ratio, two binary variables). Effect size values were transformed to $Z$ s to place all estimates on a common scale and to be consistent with similar meta-analyses [20]. A $Z$-value of 0 indicated that SES had no association with inflammation. A positive $Z$-value indicated that lower SES was associated with higher levels of inflammation. A negative $Z$-value indicated that lower SES was associated with lower levels of inflammation. The $95 \%$ confidence interval (CI) for $Z$ represents the relative precision of the measurement. Given that the available $N$ for a particular effect size may have been lower than the total $N$ in the study (e.g., due to inflammation not being assessed in all participants), estimates of effect size weight to calculate the $95 \%$ CI were based on the $N$ from which the effect size was derived. These procedures produced 63 total effect sizes from 43 eligible studies. For analyses of SES broadly defined, 35 studies were included for CRP and 18 studies were included for IL-6.

\section{Moderator analyses}

When heterogeneous effect sizes were detected, we tested whether important demographic and methodological factors, selected on the basis of prior meta-analytic work in this area and recommended control variables for studies examining inflammation [21], moderated the observed association between SES and inflammation. The following characteristics of each sample were coded: average age (examined linearly and categorically [i.e., under 18 years vs. 18 years or older]); gender composition ( $\%$ male); racial diversity (\% White) and (\% Black); and mean body mass index (BMI). Methodological characteristics of each study were coded as follows: whether SES was a continuous or binary variable; if binary, was an extreme group approach used (e.g., lowest quintile of income compared with the highest quintile); developmental period of SES assessment (e.g., childhood vs. adulthood); whether SES was a focus of the study (vs. a covariate in analyses pertaining to another topic); whether the SES measure was subjective (vs. objective); year in print; covariate present in analysis (Yes/No: age, sex, race/ethnicity, BMI, cigarette smoking, chronic health conditions, and medication use); sample source (i.e., community sample vs. population- 
based sample); study design (i.e., retrospective vs. cross-sectional vs. longitudinal); inflammatory source (i.e., plasma vs. serum vs. blood spots); transformation of inflammatory marker before analysis (i.e., to adjust for skew); if the inflammatory marker was treated as a continuous or binary variable (see Table 1 and Supplementary Material).

Random-effects models were constructed using the $Z$ estimate and the lower and upper bounds for the 95\% CI. Publication bias was assessed via Egger's [22] and Begg's [23] tests. Heterogeneity of effect sizes was estimated using the standard Cochran's $Q$ Test, which approximates a chi-square distribution with $k-1$ degrees of freedom, where $k$ is the number of effect sizes and indicates the degree of consistency of findings across studies. When the $p$ value associated with the $Q$ statistic was $\unlhd \mathbf{0 . 1 0}$, indicating heterogeneity in the effect sizes, pre-specified random-effects meta-regression moderator analyses were conducted to determine whether the study characteristics described above could explain variability across studies. We tested each moderator separately using simple regressions (i.e., the metareg command). Analyses were performed using STATA 14.2 (StataCorp, 2015).

\section{Results}

Table 1 and Supplemental Table 1 provide descriptive information for each study, including demographic and methodological moderators coded and outcomes obtained.

\section{SES and CRP}

We first examined SES among all studies that included a measure of this construct in relation to CRP levels. Among these, $Z$ s ranged from -0.01 to 0.49 (see Fig. 2). The random-effects meta-analysis found that lower SES was associated with higher CRP ( $Z=$ $0.12 ; 95 \%$ CI, 0.09-0.16) and significantly differed from zero, $Z=7.32, p<0.001$. Significant heterogeneity was observed across studies $\left(Q(34)=747.01, p<0.001 ; I^{2}=\right.$ 95\%). The bias statistic from both Egger's and Begg's publication bias tests was not significant $(p>0.10)$.

Within studies that used uniform definitions of SES based on income or education attainment, we found a similar magnitude of association between lower income and lower education and higher CRP ( $Z=0.11$ for income; $Z=0.12$ for education; both $p<0.05$; see Supplementary Material).

\section{SES and IL-6}

We also examined SES among all studies that included a measure of this construct in relation to IL-6. Among these, $Z \mathrm{~s}$ ranged from 0.08 to 0.32 (see Fig. 3). The random-effects meta-analysis found that lower SES was associated with higher IL-6 $(Z=0.15 ; 95 \% \mathrm{CI}$, $0.12-0.18)$ and significantly differed from zero, $Z=9.50, p<0.001$. There was evidence of significant heterogeneity across studies $\left(Q(17)=36.89, p=0.003 ; I^{2}=54 \%\right)$. The bias statistic from both Egger's and Begg's publication bias tests was not significant $(p>0.10)$.

Again, within studies that used uniform definitions of SES based on income or education attainment, we found a similar magnitude of association between lower income and lower 
education and higher IL-6 ( $Z=0.12$ for income; $Z=0.11$ for education; both $p<0.05$; see Supplementary Material).

\section{Moderators of the association between SES and CRP}

We explored whether the moderators described previously were associated with heterogeneity of effect sizes for the analyses with significant $Q$ statistics (see Supplementary Material for full moderator results). For the association between SES and CRP, four moderators were significantly associated with variation in effect sizes: (1) whether SES was treated as a continuous or binary variable [Coef. $=0.10, \mathrm{SE}=0.04, t=-2.45, p=0.020$ ]; (2) whether BMI was included as a covariate in analyses [Coef. $=-0.11, \mathrm{SE}=0.05, t=-2.38, p$ $=0.023$ ]; (3) whether cigarette smoking was included as a covariate in analyses [Coef. $=$ $-0.13, \mathrm{SE}=0.04, t=-2.97, p=0.005]$; and (4) whether CRP values were transformed prior to analysis [Coef. $=0.08, \mathrm{SE}=0.04, t=2.06, p=0.047]$. Meta-analyses within each set of studies indicated that the estimated effect size among those that used a binary assessment of SES was half as large $(Z=0.09 ; 95 \% \mathrm{CI}, 0.06-0.12 ; n=26)$ compared with those that used a continuous assessment of SES $(Z=0.20 ; 95 \% \mathrm{CI}, 0.11-0.28 ; n=9)$, though both produced effect sizes that were significantly different from zero ( $p s<0.001)$. Studies that did not covary for BMI $(Z=0.15 ; 95 \% \mathrm{CI}, 0.11-0.19 ; n=29)$ had a larger estimated effect size than those that $\operatorname{did}(Z=0.03 ; 95 \% \mathrm{CI},-0.01-0.06 ; n=5)$, and only those that did not covary for BMI produced an effect size that significantly differed from zero $(p<0.001)$. Studies that did not covary for cigarette smoking $(Z=0.15 ; 95 \% \mathrm{CI}, 0.11-0.18 ; n=30)$ had a larger estimated effect size than those that $\operatorname{did}(Z=0.01 ; 95 \% \mathrm{CI},-0.01-0.04 ; n=5)$, and only those that did not covary for smoking produced an effect size that significantly differed from zero $(p<0.001$ ). Analyses comparing studies that did and did not transform CRP showed larger estimated effect sizes among studies that transformed CRP prior to analyses $(Z=$ $0.15 ; 95 \%$ CI, $0.10-0.20 ; n=23)$ compared with those that did not transform $(Z=0.07 ; 95 \%$ CI, $0.02-0.12 ; n=12)$, though both produced effect sizes that were significantly different from zero $(p s<0.01)$.

\section{Moderators of the association between SES and IL-6}

Turning to SES and IL-6, four moderators predicted significant variability in effect sizes: (1) year in print (Coef. $=0.01, \mathrm{SE}=0.003, t=2.62, p=0.019)$; (2) whether SES was a subjective or objective assessment (Coef. $=0.18, \mathrm{SE}=0.08, t=2.23, p=0.041$ ); (3) whether age was included as a covariate in analyses (Coef. $=-0.07, \mathrm{SE}=0.02, t=-2.99, p=0.009$ ); and (4) whether IL-6 levels were transformed prior to analysis (Coef. $=0.07, \mathrm{SE}=0.02, t=$ $2.79, p=0.013)$. Greater effect sizes were found among studies published more recently. Only one study included used a subjective measure of SES [24]. With it excluded, the estimated effect size that was just slightly smaller than the full set of studies $(Z=0.14 ; 95 \%$ CI, $0.11-0.17 ; n=17)$, and produced an effect size that was significantly different from zero $(p<0.001)$. Studies that did not covary for age in analyses $(Z=0.16 ; 95 \%$ CI, $0.13-0.19 ; n$ $=13)$ had larger effect sizes than those that $\operatorname{did}(Z=0.11 ; 95 \% \mathrm{CI}, 0.07-0.15 ; n=5)$, and both produced effect sizes that were significantly different from zero ( $p$ s $<0.001$ ). Lastly, studies that transformed IL-6 $(Z=0.16 ; 95 \% \mathrm{CI}, 0.13-0.19)$ demonstrated a stronger association than those studies that did not transform IL-6 ( $Z=0.10 ; 95 \% \mathrm{CI}, 0.06-0.15 ; n=$ $4)$, though both sets significantly differed from zero ( $p$ s $<0.001)$. 


\section{Discussion}

In the first known meta-analysis to examine the association between SES and levels of systemic inflammation, we quantified the effects from 43 published papers comprising of over 110,000 participants, yielding 63 relevant effect sizes. These analyses produced several key findings. First, we found a significant association between SES, broadly defined, and circulating levels of both CRP and IL-6, such that individuals with lower SES showed higher levels of these inflammatory markers. This is notable given that elevated levels of systemic inflammation are a risk factor for disease development and psychopathology [8, 10,11], and yet studies included in the meta-analysis excluded samples selected on the basis of any mental or physical health problem. In other words, these data suggest the possibility that low SES may set individuals on a pro-inflammatory trajectory that can be observed in the general population rather than only among individuals currently experiencing a chronic disease. Second, analyses among studies using narrower definitions of SES (i.e., income and educational attainment) revealed remarkably similar, significant effects of both factors relating to CRP and IL-6. Indeed, the 95\% CIs of the effect sizes for the associations between income and CRP, income and IL-6, education and CRP, and education and IL-6 were all overlapping, indicating no statistically significant differences between the magnitude of these associations. Thus, this study provides evidence that despite potential meaningful differences in these indices [25], both low income and low levels of education are related to higher levels of systemic inflammation. Finally, there was significant heterogeneity in effects across studies, and moderator analyses shed some light on important factors that may contribute to this heterogeneity, which we expand upon below. Taken together, these meta-analytic results indicate that there is a consistent association, albeit on average a small effect, between SES and inflammatory markers of risk for negative outcomes. Though the evidence is not causal, our findings provide support for the notion that upregulation of inflammatory processes may play a part in contributing to known socioeconomic disparities in disease morbidity and psychopathology.

In contextualizing the magnitude of the relationship between SES and inflammatory markers observed here, it is useful to consider the effects observed in other recent meta-analyses that have examined the association between psychosocial/behavioral factors and inflammation. For example, the estimated overall weighted association between SES and inflammatory markers ( $Z=0.12$ and $Z=0.15$ for CRP and IL-6, respectively) appears somewhat stronger than the association found for childhood trauma and inflammation $(Z=0.10$ and 0.08 for CRP and IL-6, respectively) [20] and similar to the effects of depression ( $d=0.15$ and 0.25 for CRP and IL-6, respectively) [26] and sleep disturbance ( $d=0.12$ and 0.20 for CRP and IL-6, respectively) [27] on inflammatory markers. Compared with these other markers of distress, SES is the least direct in its impact, yet the association between SES and systemic inflammation is similar in strength, suggesting that SES may be an important contributor to levels of inflammation.

While the present meta-analysis provides greater confidence in the association between SES and inflammation, this approach is not designed to test why low SES is associated with inflammation. Moderator analyses may provide some insight, however, as studies that controlled for BMI and/or cigarette smoking showed a much smaller association between 
SES and CRP than those that did not control for these factors (which was not found for analyses examining IL-6). Obesity and smoking are two possible mediators, or mechanisms, linking SES and levels of CRP, as the SES-CRP link is attenuated if SES-related differences in obesity and smoking are eliminated (though other factors are likely important as well [28]). This result is consistent with another recent meta-analysis in this area [29], which also found that BMI explained significant variability in the association between childhood SES and adult CRP. Taken together, these findings provide further support for the usefulness of population health initiatives aimed at weight management and smoking cessation to help reduce health disparities across the SES spectrum [30, 31], and targeted prevention programs may be useful in reducing elevated CRP among those at highest risk.

In addition to providing insights important for clinicians and public health professionals, results of moderator analyses can also be used to guide decisions for future research examining the relation between SES and inflammation. For example, we observed stronger effect sizes for studies that transformed CRP and IL-6 before analysis to account for the skew in such data, suggesting that using non-transformed inflammatory marker data may lead to underestimations of their relation with SES. This is perhaps due to the fact that the use of untransformed data does not meet statistical assumptions of normality, thus possibly producing biased estimates. Further, for studies examining the relation between SES and CRP, we observed stronger effect sizes among studies that treated SES as a continuous measure compared with those that used a binary SES measure (e.g., comparing those with and without a high school degree). This result suggests that levels of CRP vary across the entire SES spectrum and not just when comparing groups, including extreme groups (e.g., comparing those who did not complete high school to those with a graduate degree). Thus, results from these moderator analyses suggest that future work should ensure adequate transformation of inflammatory data prior to analysis and should conceptualize SES as a continuous measure (particularly among studies using CRP) [1].

Turning to IL-6, we found that year in print, whether age was controlled for analyses and whether SES was a subjective or objective measure, moderated the magnitude of the association between SES and IL-6; with most recent papers, effects that did not control for age, and subjective SES measures, respectively, yielding stronger associations. While moderator analyses are useful, the lack of consistency of moderator variables for analyses examining CRP versus IL-6 are interesting to note but difficult to interpret, as the difference may reflect true variation in the nature between SES and each marker, or perhaps differences related to the studies that provided each marker, as there were more studies that examined CRP than IL-6. Further, CRP is generally a more stable indicator of chronic inflammatory activation [32] and thus may be more strongly related to behavioral factors like smoking and BMI. Moving forward, examination of the moderators identified here in large epidemiological studies of a single cohort that assesses both CRP and IL-6 would be most informative about the potential specificity of each moderator and to gain confidence in the patterns observed here.

We did not find evidence that the magnitude of the association between SES and CRP or IL-6 was moderated by a number of demographic and study design factors that are thought to be important for research in this area (e.g., sex, race, age, and BMI makeup of the sample, 
SES in childhood vs. adulthood, if inflammation was measured in pediatric or adult samples, type of study design, sample recruitment strategy, source of inflammation for assay, and if inflammation was measured on a continuum or with a cutoff). It will be important for future work in this area to continue to measure these factors and examine if the association between SES and inflammation exists for all individuals and across all types of studies, given the limited ability for broad study-level patterns to inform individual-level mechanistic processes.

A number of limitations of this study should be noted. First, only studies that included nonpatient samples were included in the analysis, so we cannot draw conclusions regarding the association between SES and inflammation in specific disease states. Second, we restricted our analysis to studies conducted with North American samples in an effort to account for the fact that socioeconomic influences on health vary strongly across countries and continents [19]. Future work could examine the association between SES and inflammation as a function of country-level economic inequality, given that inequality has shown to influence health over-and-above individual-level SES factors [33]. Third, our subanalyses focused on income and educational attainment, given that these were the most commonlyassessed measures of SES in this literature. However, there is growing appreciation of the role that neighborhood-level SES [34], as well as subjective perceptions of SES [35], may play in influencing health; as more studies accumulate in this area, we will be able to examine the association between these (and other) alternative indicators of SES and their associations with inflammation. Fourth, we undoubtedly missed studies in which SES and inflammation were examined, as many studies include SES as a covariate, and these may not have been surfaced in our search strategy. Importantly, however, we found no difference in studies in which SES was a primary focus or merely a covariate. Further, we only examined the association between SES and CRP/IL-6, and thus cannot speak to the magnitude of the relationship between SES and other markers of inflammation (e.g., TNF-a). Finally, due to inconsistencies in the literature in the use, conceptualization, and measurement of psychological stress [36], we did not explore stress as a possible moderator of the link between SES and inflammation. This would be a valuable contribution for future metaanalyses in this area.

In sum, there is a significant association between SES and levels of inflammation (both CRP and IL-6). These results are important for our understanding of how social inequalities may become health inequalities. As next steps, it will be important to explore mechanistic pathways (i.e., psychological and biological) by which SES may influence inflammation. Results from moderator analyses presented here suggest that the association between SES and CRP may be explained in-part by SES disparities in obesity and smoking, demonstrating the importance of behavioral processes in contributing to this link. In addition, researchers should consider building upon theoretical foundations to explore the potential pathways of psychosocial stress, social comparison, and scarcity perceptions, as well as the more proximal biological mechanisms that may link SES and inflammation (e.g., sympathetic nervous system activation). Interventions that improve SES can be leveraged to test causal assumptions about the effect of SES on inflammatory markers. Such mechanistic and causal insights would allow for directed interventions at a population level with the potential to improve mental and physical health for all individuals, regardless of SES background. 


\section{Supplementary Material}

Refer to Web version on PubMed Central for supplementary material.

\section{Acknowledgements}

K.L.H. is funded by the National Institutes of Health (F32 MH107129), the Brain and Behavior Research Foundation (Young Investigator Award 23819), Klingenstein Third Generation Foundation, and a Jacobs Foundation Early Career Research Fellowship. The authors thank Lillian Dillard for her assistance with literature searches and coding; and Allison Appleton, Jennifer Morozink Boylan, Ben Chapman, Edith Chen, Yvette Cozier, Ari Elliot, Andrew Fuligni, Neha John-Henderon, Anne-marie Koster, Thomas McDade, Gregory Miller, Markus Schafer, Kammi Schmeer, and Hannah Schreier for providing relevant data and information from their published papers on SES and inflammation.

\section{References}

1. Adler NE, Boyce T, Chesney MA, Cohen S, Folkman S, Kahn RL, et al. Socioeconomic status and health: the challenge of the gradient. Am Psychol. 1994;49:15-24. 10.1037/0003-066X.49.1.15 [PubMed: 8122813]

2. Marmot MG. Status syndrome: a challenge to medicine. J Am Med Assoc. 2006;295:1304-7. 10.1001/jama.295.11.1304

3. Lorant V, Deliège D, Eaton W, Robert A, Philippot P, Ansseau M. Socioeconomic inequalities in depression: a meta-analysis. Am J Epidemiol. 2003;157:98-112. 10.1093/aje/kwf182 [PubMed: 12522017]

4. Agerbo E, Sullivan PF, Vilhjálmsson BJ, et al. Polygenic risk score, parental socioeconomic status, family history of psychiatric disorders, and the risk for schizophrenia: a Danish population-based study and meta-analysis. JAMA Psychiatry. 2015;72:635-41. 10.1001/jamapsychiatry.2015.0346 [PubMed: 25830477]

5. Clark AM, DesMeules M, Luo W, Duncan AS, Wielgosz A. Socioeconomic status and cardiovascular disease: risks and implications for care. Nat Rev Cardiol. 2009;6:712-22. 10.1038/ nrcardio.2009.163 [PubMed: 19770848]

6. Everson SA, Maty SC, Lynch JW, Kaplan GA. Epidemiologic evidence for the relation between socioeconomic status and depression, obesity, and diabetes. J Psychosom Res. 2002;53:891-5. [PubMed: 12377299]

7. Shishehbor MH. Association of socioeconomic status with functional capacity, heart rate recovery, and all-cause mortality. JAMA. 2006;295:784 10.1001/jama.295.7.784 [PubMed: 16478901]

8. Gimeno D, Kivimäki M, Brunner EJ, et al. Associations of C-reactive protein and interleukin-6 with cognitive symptoms of depression: 12-year follow-up of the Whitehall II study. Psychol Med. 2009;39:413 10.1017/S0033291708003723 [PubMed: 18533059]

9. Davidson KW, Schwartz JE, Kirkland SA, et al. Relation of inflammation to depression and incident coronary heart disease (from the Canadian Nova Scotia Health Survey [NSHS95] Prospective Population Study). Am J Cardiol. 2009;103:755-61. 10.1016/j.amjcard.2008.11.035 [PubMed: 19268727]

10. Rost NS, Wolf PA, Kase CS, et al. Plasma concentration of C-reactive protein and risk of ischemic stroke and transient ischemic attack: the Framingham study. Stroke. 2001;32:2575-9. 10.1161/ HS1101.098151 [PubMed: 11692019]

11. Möhlenkamp S, Lehmann N, Moebus S, et al. Quantification of coronary atherosclerosis and inflammation to predict coronary events and all-cause mortality. J Am Coll Cardiol. 2011;57:1455-64. 10.1016/j.jacc.2010.10.043 [PubMed: 21435514]

12. Cohen S, Janicki-Deverts D, Miller GE. Psychological stress and disease. JAMA. 2007;298:16857. 10.1001/jama.298.14.1685 [PubMed: 17925521]

13. Mora S, Lee I-M, Buring JE, Ridker PM. Association of physical activity and body mass index with novel and traditional cardiovascular biomarkers in women. JAMA. 2006;295:1412-9. http:// jama.ama-assn.org/cgi/content/abstract/295/12/1412 [PubMed: 16551713] 
14. Shiels MS, Katki HA, Freedman ND, et al. Cigarette smoking and variations in systemic immune and inflammation markers. J Natl Cancer Inst. 2014;106:1-8. 10.1093/jnci/dju294

15. Woodward M, Rumley A, Tunstall-Pedoe H, Lowe GDO. Associations of blood rheology and interleukin-6 with cardiovascular risk factors and prevalent cardiovascular disease. Br J Haematol. 1999;104:246-57. 10.1046/j.1365-2141.1999.01158.x [PubMed: 10050704]

16. Ma Mendall, Patel P, Asante M, et al. Relation of serum cytokine concentrations to cardiovascular risk factors and coronary heart disease. Heart. 1997;78:273-7. 10.1136/hrt.78.3.273 [PubMed: 9391290]

17. Steptoe A, Owen N, Kunz-Ebrecht S, Mohamed-Ali V. Inflammatory cytokines, socioeconomic status, and acute stress responsivity. Brain Behav Immun. 2002;16:774-84. 10.1016/ S0889-1591(02)00030-2 [PubMed: 12480506]

18. Ford ES. Does exercise reduce inflammation? Physical activity and C-reactive protein among U.S. adults. Epidemiology. 2002;13:561-8. 10.1097/01.EDE.0000023965.92535.C0 [PubMed: 12192226]

19. Marmot M Social determinants of health inequalities. Lancet. 2005;365:1099-104. 10.1016/ S0140-6736(05)71146-6 [PubMed: 15781105]

20. Baumeister D, Akhtar R, Ciufolini S, Pariante CM, Mondelli V. Childhood trauma and adulthood inflammation: a meta-analysis of peripheral C-reactive protein, interleukin-6 and tumour necrosis factor-a. Mol Psychiatry. 2016;21:642-9. 10.1038/mp.2015.67 [PubMed: 26033244]

21. O'Connor M-F, Bower JE, Jin H, et al. To assess, to control, to exclude: effects of biobehavioral factors on circulating inflammatory markers. Brain Behav Immun. 2009;23:887-97. 10.1016/ j.bbi.2009.04.005 [PubMed: 19389469]

22. Egger M, Davey Smith G, Schneider M, Minder C. Bias in meta-analysis detected by a simple, graphical test. BMJ. 1997;315:629-34. 10.1136/BMJ.315.7109.629 [PubMed: 9310563]

23. Begg CB, Mazumdar M. Operating characteristics of a rank correlation test for publication bias. Source: Biom. 1994;50:1088-101.

24. John-Henderson N, Jacobs EG, Mendoza-Denton R, Francis DD. Wealth, health, and the moderating role of implicit social class bias. Ann Behav Med. 2013;45:173-9. 10.1007/ s12160-012-9443-9 [PubMed: 23229159]

25. Duncan GJ, Magnuson K. Socioeconomic status and cognitive functioning: moving from correlation to causation. Wiley Interdiscip Rev Cogn Sci. 2012;3:377-86. 10.1002/wcs.1176 [PubMed: 26301469]

26. Howren MB, Lamkin DM, Suls J. Associations of depression with C-reactive protein, IL-1, and IL-6: a meta-analysis. Psychosom Med. 2009;71:171-86. 10.1097/PSY.0b013e3181907c1b [PubMed: 19188531]

27. Irwin MR, Olmstead R, Carroll JE. Sleep disturbance, sleep duration, and inflammation: a systematic review and meta-analysis of cohort studies and experimental sleep deprivation. Biol Psychiatry. 2016;80:40-52. 10.1016/j.biopsych.2015.05.014 [PubMed: 26140821]

28. Lantz PM, House JS, Lepkowski JM, Williams DR, Mero RP, ChenJ. Socioeconomic factors, health behaviors, and mortality prospective study of US adults. J Am Med Assoc. 1998;279:17038.

29. Liu RS, Aiello AE, Mensah FK, et al. Socioeconomic status in childhood and C reactive protein in adulthood: a systematic review and meta-analysis. J Epidemiol Community Heal. 2017;71:817-26. 10.1136/jech-2016-208646

30. Wang Y, Beydoun MA. The obesity epidemic in the United States -Gender, age, socioeconomic, racial/ethnic, and geographic characteristics: A systematic review and meta-regression analysis. Epidemiol Rev. 2007;29:6-28. 10.1093/epirev/mxm007 [PubMed: 17510091]

31. Hiscock R, Bauld L, Amos A, Fidler JA, Munafò M. Socioeconomic status and smoking: a review. Ann N Y Acad Sci. 2012;1248:107-23. 10.1111/j.1749-6632.2011.06202.x [PubMed: 22092035]

32. Gabay C, Kushner I. Acute-phase proteins and other systemic responses to inflammation. N Engl J Med. 1999;340:448-54. [PubMed: 9971870]

33. Wilkinson R, Pickett K. The spirit level: why more equal societies alomost always do better. Nurs Stand. 2009;12:14 10.1080/15700763.2011.577928 
34. Sampson RJ, Morenoff JD, Gannon-Rowley T. Assessing "Neighborhood Effects": social processes and new directions in research. Annu Rev Sociol. 2002;28:443-78. 10.1146/ annurev.soc.28.110601.141114

35. Operario D, Adler NE, Williams DR. Subjective social status: reliability and predictive utility for global health. Psychol Heal. 2004;19:237-46. 10.1080/08870440310001638098

36. Epel ES, Crosswell AD, Mayer SE. et al. More than a feeling:a unified view of stress measurement for population science. Front Neuroendocrinol. 2018;49:146-69. 10.1016/j.yfrne.2018.03.001. [PubMed: 29551356]

37. Appleton AA, Buka SL, McCormick MC, Koenen KC, Loucks EB, Kubzansky LD. The association between childhood emotional functioning and adulthood inflammation is modified by early-life socioeconomic status. Heal Psychol. 2012;31:413-22. 10.1037/a0027300

38. Boylan JM, Ryff CD. Varieties of anger and the inverse link between education and inflammation: toward an integrative framework. Psychosom Med. 2013;75:566-74. 10.1097/ PSY.0b013e31829683bd [PubMed: 23766379]

39. Broyles ST, Staiano AE, Drazba KT, Gupta AK, Sothern M, Katzmarzyk PT. Elevated C-reactive protein in children from risky neighborhoods: evidence for a stress pathway linking neighborhoods and inflammation in children. PLoS ONE 2012;7:1-9. 10.1371/journal.pone.0045419.

40. Carroll JE, Cohen S, Marsland AL. Early childhood socioeconomic status is associated with circulating interleukin-6 among mid-life adults. Brain Behav Immun. 2011;25:1468-74. 10.1016/ j.bbi.2011.05.016 [PubMed: 21672624]

41. Chapman BP, Khan A, Harper M, et al. Gender, race/ethnicity, personality, and interleukin-6 in urban primary care patients. Brain Behav Immun. 2009;23:636-42. 10.1016/j.bbi.2008.12.009 [PubMed: 19162168]

42. Chiang JJ, Bower JE, Almeida DM, Irwin MR, Seeman TE, Fuligni AJ. Socioeconomic status, daily affective and social experiences, and inflammation during adolescence. Psychosom Med. 2015;77:256-66. 10.1097/PSY.0000000000000160 [PubMed: 25829237]

43. Clark CR, Ridker PM, Ommerborn MJ, et al. Cardiovascular inflammation in healthy women: multilevel associations with state-level prosperity, productivity and income inequality. BMC Public Health. 2012;12:211 10.1186/1471-2458-12-211 [PubMed: 22433166]

44. Cole SW, Arevalo JMG, Manu K, et al. Antagonistic pleiotropy at the human IL6 promoter confers genetic resilience to the proinflammatory effects of adverse social conditions in adolescence. Dev Psychol. 2011;47:1173-80. 10.1037/a0023871 [PubMed: 21639625]

45. Cozier YC, Albert MA, Castro-Webb N, et al. Neighborhood socioeconomic status in relation to serum biomarkers in the black women's health study. J Urban Health. 2016;93:279-91. 10.1007/ s11524-016-0034-0 [PubMed: 27000125]

46. Cushman M, McClure LA, Howard VJ, Jenny NS, Lakoski SG, Howard G. Implications of increased C-reactive protein for cardiovascular risk stratification in black and white men and women in the US. Clin Chem. 2009;55:1627-36. 10.1373/clinchem.2008.122093 [PubMed: 19643839]

47. Dowd JB, Haan MN, Blythe L, Moore K, Aiello AE. Socioeconomic gradients in immune response to latent infection. Am J Epidemiol. 2008;167:112-20. 10.1093/aje/kwm247 [PubMed: 17873099]

48. Dowd JB, Zajacova A, Aiello AE. Predictors of inflammation in U.S. children aged 316 years. Am J Prev Med. 2010;39:314-20. 10.1016/j.amepre.2010.05.014 [PubMed: 20837281]

49. Elliot AJ, Chapman BP. Socioeconomic status, psychological resources, and inflammatorymarkers: results from the MIDUS study. Heal Psychol. 2016;35:1205-13. 10.1037/hea0000392

50. Fedewa MV, Das BM, Forehand RL, Evans EM. Area-level socioeconomic status, adiposity, physical activity, and inflammation in young adults, 2013. Prev Chronic Dis. 2014;11:E130 10.5888/pcd11.140090 [PubMed: 25078567]

51. Gallo LC, Fortmann AL, de Los Monteros KE, et al. Individual and neighborhood socioeconomic status and inflammation in Mexican American women: what is the role of obesity? Psychosom Med. 2012;74:535-42. 10.1097/PSY.0b013e31824f5f6d [PubMed: 22582313]

52. Herd P, Karraker A, Friedman E. The social patterns of a biological risk factor for disease: race, gender, socioeconomic position, and C-reactive protein. J Gerontol B Psychol Sci Soc Sci. 2012;67:503-13. 10.1093/geronb/gbs048 [PubMed: 22588996] 
53. Hostinar CE, Ross KM, Chen E, Miller GE. Modeling the association between lifecourse socioeconomic disadvantage and systemic inflammation in healthy adults: The role of self-control. Heal Psychol. 2015;34:580-90. 10.1037/hea0000130.

54. Janicki-Deverts D, Cohen S, Matthews KA, Cullen MR. History of unemployment predicts future elevations in C-reactive protein among male participants in the coronary artery risk development in young adults (CARDIA) study. Ann Behav Med. 2008;36:176-85. 10.1007/s12160-008-9056-5 [PubMed: 18784972]

55. Joseph J, Depp C, Martin AS, et al. Associations of high sensitivity C-reactive protein levels in schizophrenia and comparison groups. Schizophr Res. 2015;168:456-60. 10.1016/ j.schres.2015.08.019 [PubMed: 26341579]

56. Koster A, Bosma H, Penninx BWJH, et al. Association of inflammatory markers with socioeconomic status. J Gerontol A Biol Sci Med Sci. 2006;61:284-90. 10.1093/gerona/61.3.284 [PubMed: 16567379]

57. Loucks EB, Sullivan LM, Hayes LJ, et al. Association of educational level with inflammatory markers in the Framingham Off-spring Study. Am J Epidemiol. 2006;163:622-8. 10.1093/aje/ kwj076 [PubMed: 16421236]

58. Loucks EB, Berkman LF, Gruenewald TL, Seeman TE. Relation of social integration to inflammatory marker concentrations in men and women 70 to 79 years. Am J Cardiol. 2006;97:1010-6. 10.1016/j.amjcard.2005.10.043 [PubMed: 16563907]

59. Marsland AL, Petersen KL, Sathanoori R, et al. Interleukin-6 covaries inversely with cognitive performance among middle-aged community volunteers. Psychosom Med. 2006;68:895-903. 10.1097/01.psy.0000238451.22174.92 [PubMed: 17132839]

60. McDade TW, Hawkley LC, Cacioppo JT. Psychosocial and behavioral predictors of inflammation in middle-aged and older adults: the Chicago health, aging, and social relations study. Psychosom Med. 2006;68:376-81. 10.1097/01.psy.0000221371.43607.64 [PubMed: 16738067]

61. Miller GE, Chen E, Fok AK, et al. Low early-life social class leaves a biological residue manifested by decreased glucocorticoid and increased proinflammatory signaling. Proc Natl Acad Sci USA. 2009;106:14716-21. 10.1073/pnas.0902971106 [PubMed: 19617551]

62. Miller GE, Chen E. Harsh family climate in early life presages the emergence of a proinflammatory phenotype in adolescence. Psychol Sci. 2010;21:848-56. 10.1177/0956797610370161 [PubMed: 20431047]

63. Muennig P, Sohler N, Mahato B. Socioeconomic status as an independent predictor of physiological biomarkers of cardiovascular disease: Evidence from NHANES. Prev Med (Baltim). 2007;45:35-40. 10.1016/j.ypmed.2007.04.005

64. Mwendwa DT, Ali MK, Sims RC, et al. Dispositional depression and hostility are associated with inflammatory markers of cardiovascular disease in African Americans. Brain Behav Immun. 2013;28:72-82. 10.1016/j.bbi.2012.10.019 [PubMed: 23123367]

65. Paalani M, Lee JW, Haddad E, Tonstad S. Determinants of inflammatory markers in a bi-ethnic population. Ethn Dis. 2011; 21:142-9. [PubMed: 21749016]

66. Paul K, Boutain D, Agnew K, Thomas J, Hitti J. The relationship between racial identity, income, stress and C-reactive protein among parous women: implications for preterm birth disparity research. J Natl Med Assoc. 2008;100:540-6. 10.1016/S0027-9684(15)31300-6 [PubMed: 18507206]

67. Petersen KL, Marsland AL, Flory J, Votruba-Drzal E, Muldoon MF, Manuck SB. Community socioeconomic status is associated with circulating interleukin-6 and C-reactive protein. Psychosom Med. 2008;70:646-52. 10.1097/PSY.0b013e31817b8ee4 [PubMed: 18606725]

68. Pietras SA, Goodman E. Socioeconomic status gradients in inflammation in adolescence. Psychosom Med. 2013;75:442-8. 10.1097/PSY.0b013e31828b871a [PubMed: 23533285]

69. Schmeer KK, Yoon AJ. Home sweet home? Home physical environment and inflammation in children. Soc Sci Res. 2016;60:236-48. 10.1016/j.ssresearch.2016.04.001 [PubMed: 27712682]

70. Schmeer KK, Yoon A. Socioeconomic status inequalities in low-grade inflammation during childhood. Arch Dis Child. 2016;101:1043-7. 10.1136/archdischild-2016-310837 [PubMed: 27371708] 
71. Schreier HMC, Chen E. Socioeconomic status in one's childhood predicts offspring cardiovascular risk. Brain Behav Immun. 2010;24:1324-31. 10.1016/j.bbi.2010.06.007 [PubMed: 20600814]

72. Schreier HMC, Roy LB, Frimer LT, Chen E. Family chaos and adolescent inflammatory profiles. Psychosom Med. 2014;76:460-7. 10.1097/PSY.0000000000000078 [PubMed: 24979577]

73. Shanahan L, Freeman J, Bauldry S. Is very high C-reactive protein in young adults associated with indicators of chronic disease risk? Psychoneuroendocrinology. 2014;40:76-85. 10.1016/ j.psyneuen.2013.10.019 [PubMed: 24485478]

74. Sin NL, Graham-Engeland JE, Ong AD, Almeida DM. Affective reactivity to daily stressors is associated with elevated inflammation. Health Psychol. 2015;34:1154-65. 10.1037/hea0000240 [PubMed: 26030309]

75. Sturgeon JA, Arewasikporn A, Okun MA, Davis MC, Ong AD, Zautra AJ. The psychosocial context of financial stress: implications for inflammation and psychological health. Psychosom Med. 2015 10.1097/PSY.0000000000000276.

76. Taylor SE, Lehman BJ, Kiefe CI, Seeman TE. Relationship of early life stress and psychological functioning to adult C-reactive protein in the coronary artery risk development in young adults study. Biol Psychiatry. 2006;60:819-24. 10.1016/j.biopsych.2006.03.016 [PubMed: 16712805]

77. Zhang X, Shu X-OO, Signorello LB, et al. Correlates of high serum C-reactive protein levels in a socioeconomically disadvantaged population. Dis Markers. 2008;24:351-9. 10.1155/2008/509138 [PubMed: 18688084] 


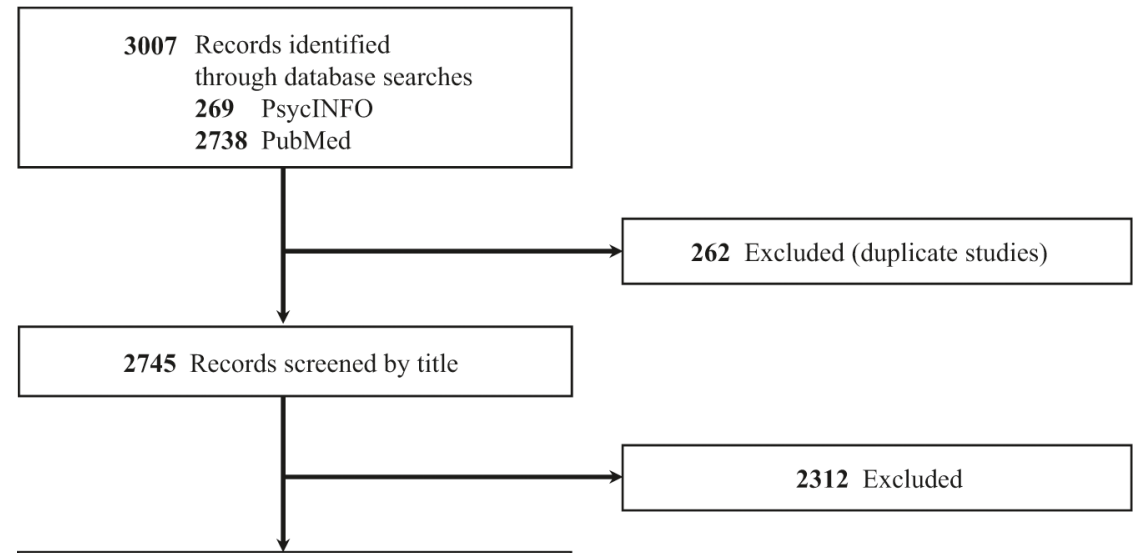

433 Records screened by abstract

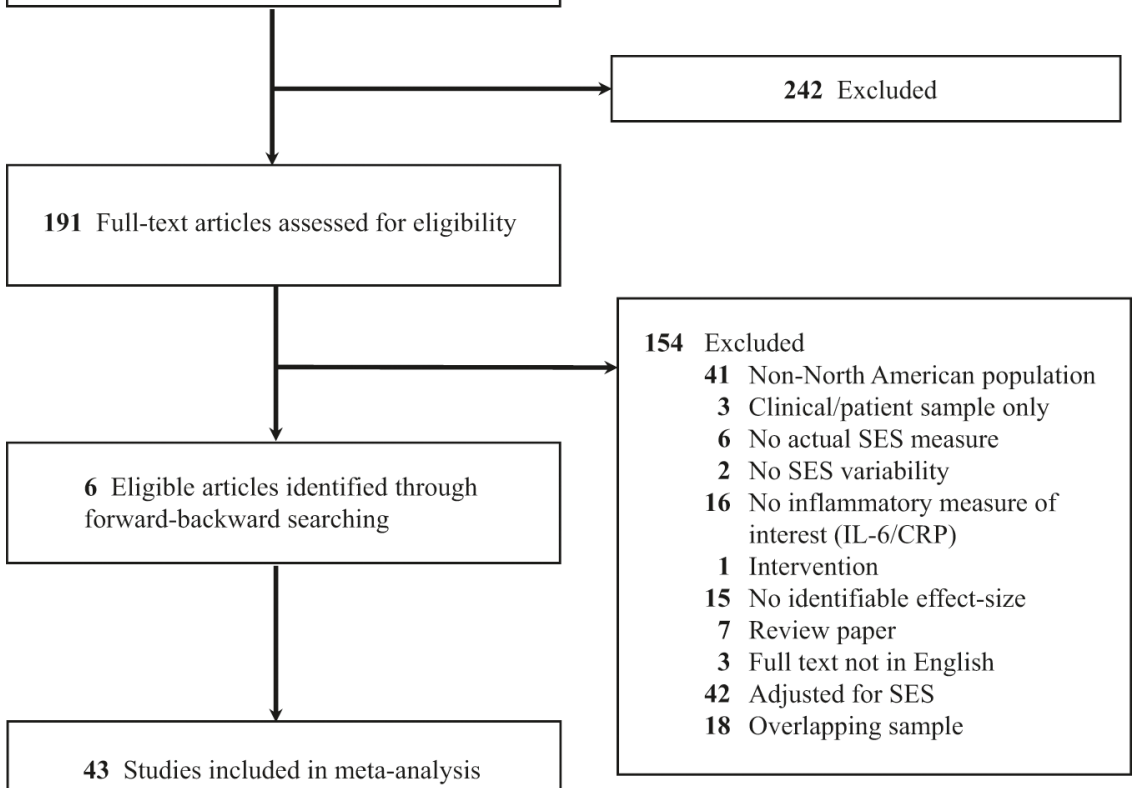

Fig. 1.

Preferred reporting items for systematic reviews and meta-analyses (PRISMA) flow diagram for identification and inclusion of studies in the meta-analysis 


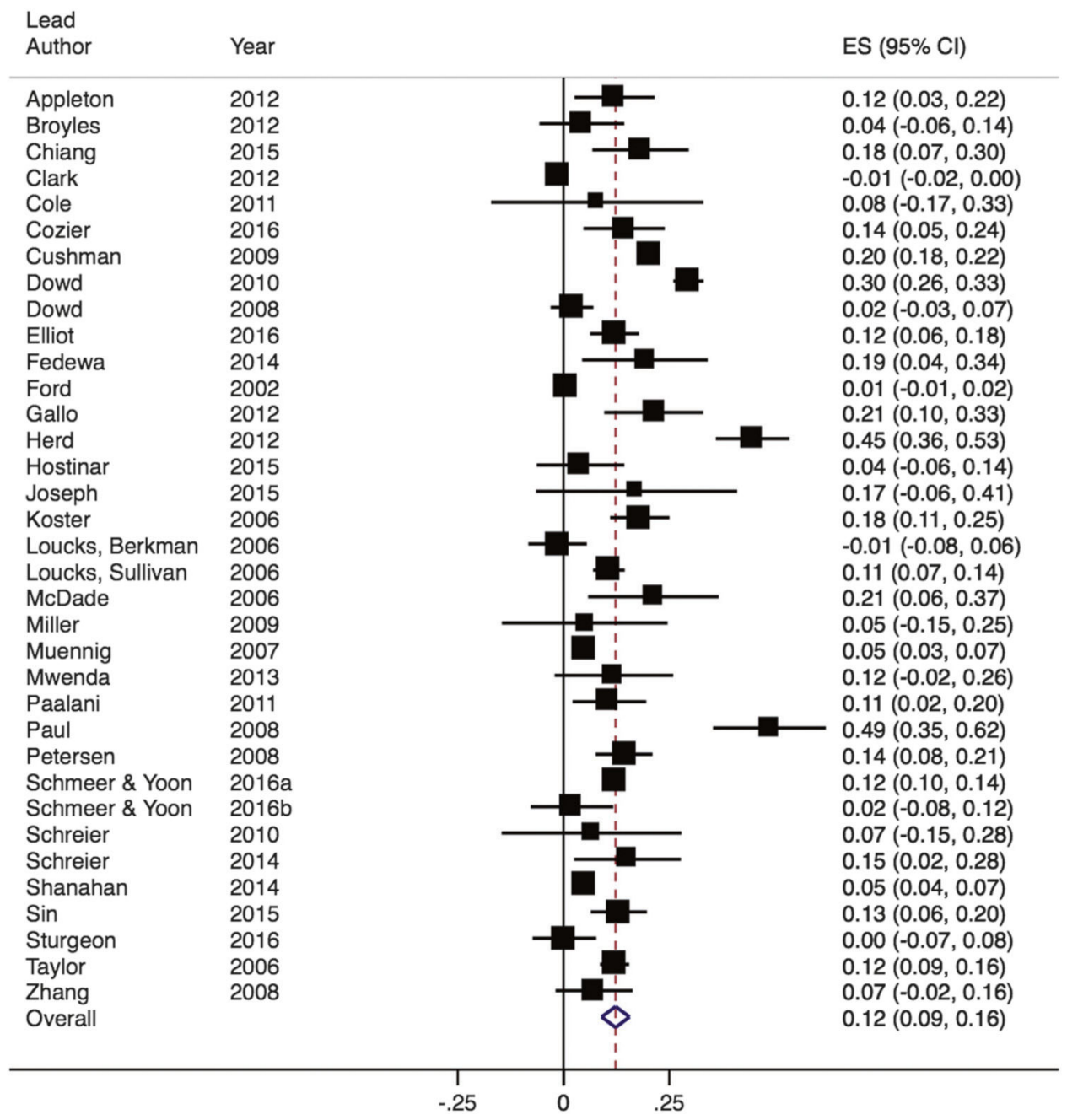

Fig. 2.

Forest plot of effect sizes from studies examining the association between SES and CRP. Box sizes are indicative of study weight, with box center positioned at the point estimate of the effect. Horizontal lines indicate 95\% CIs. Dashed vertical line indicates the estimated meta-analytic effect 


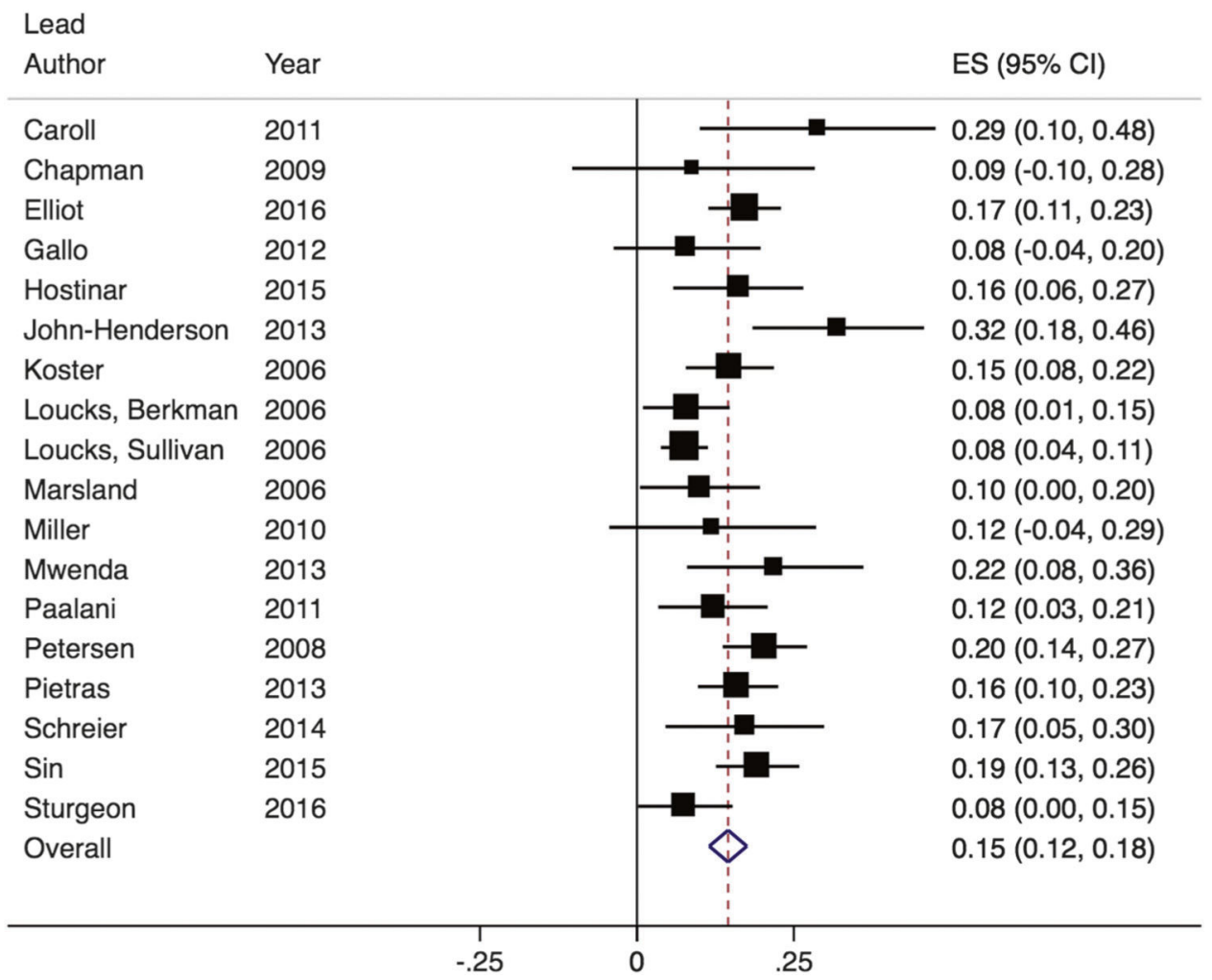

Fig. 3.

Forest plot of effect sizes from studies examining the association between SES and IL-6. Box sizes are indicative of study weight, with box center positioned at the point estimate of the effect. Horizontal lines indicate 95\% CIs. Dashed vertical line indicates the estimated meta-analytic effect 


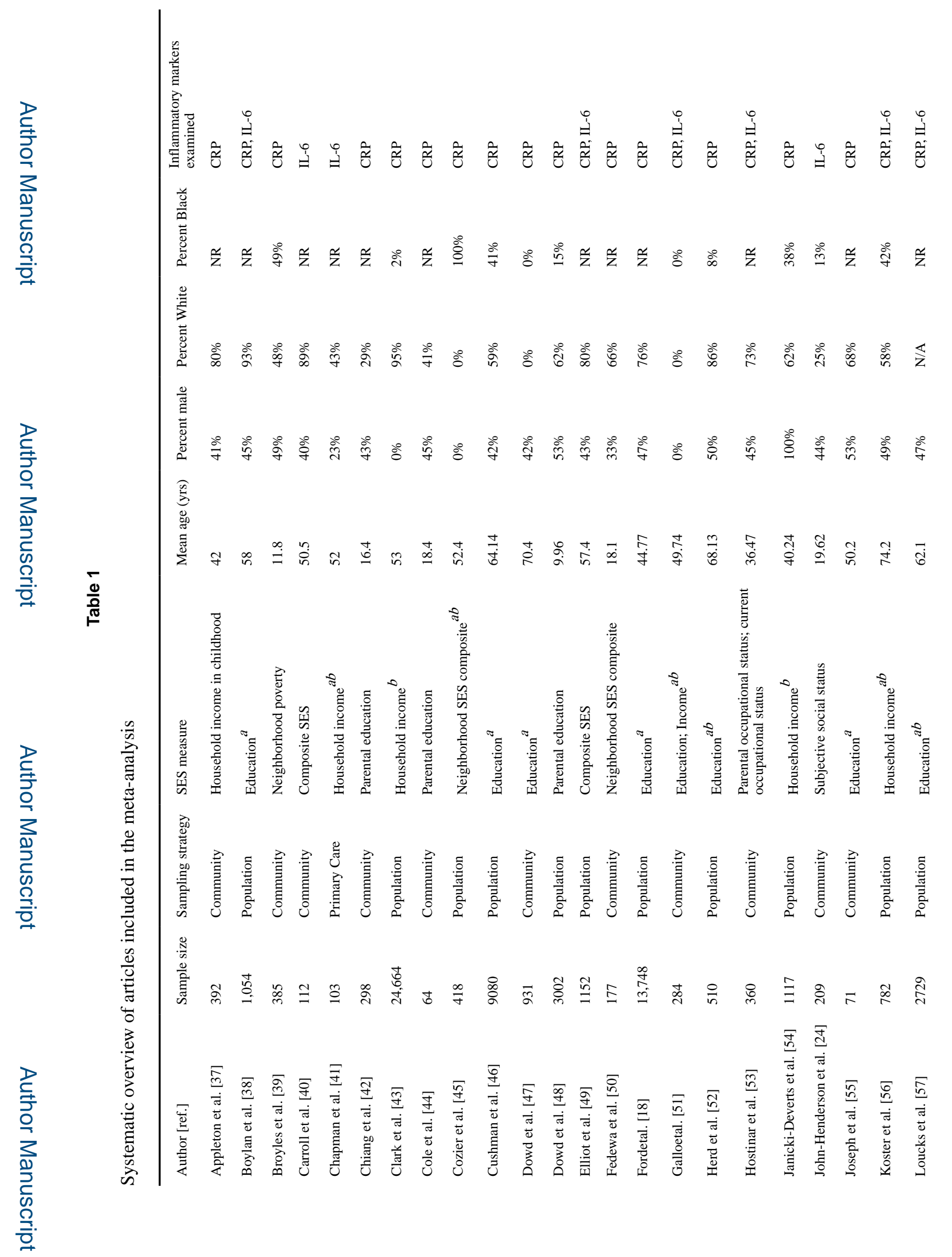

Mol Psychiatry. Author manuscript; available in PMC 2020 April 19. 


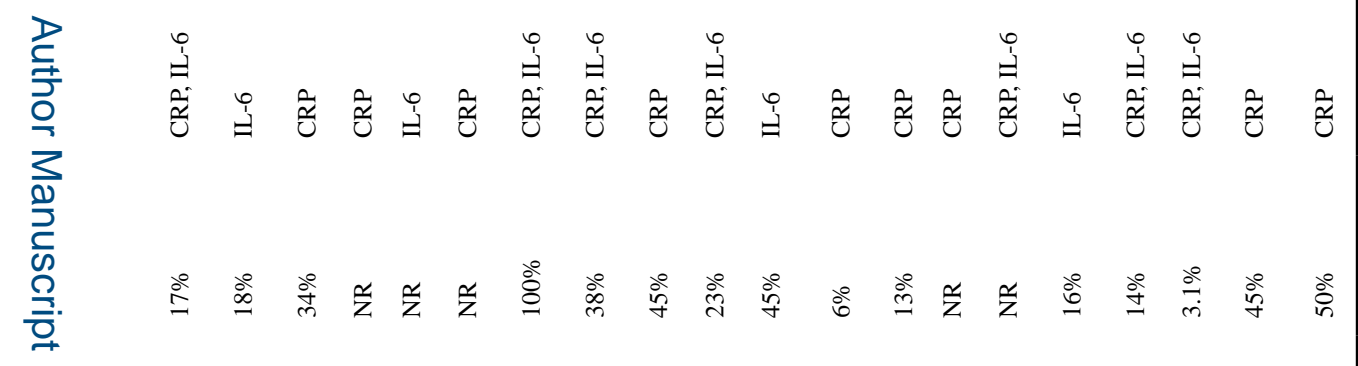

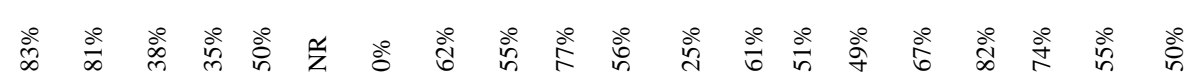

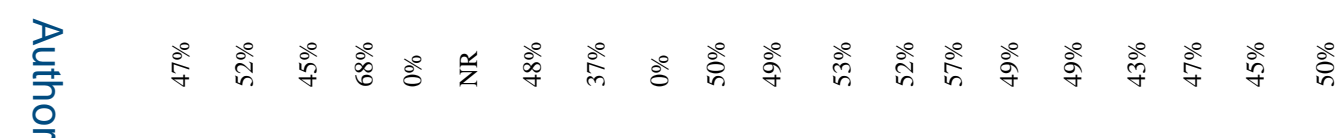

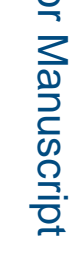

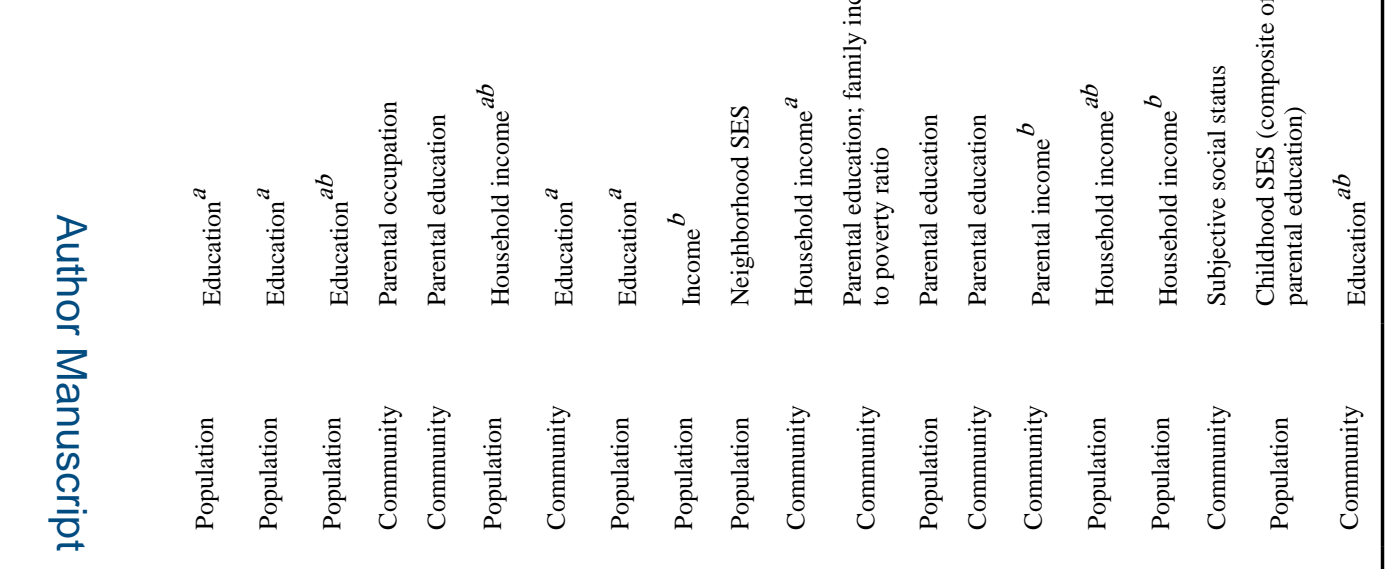

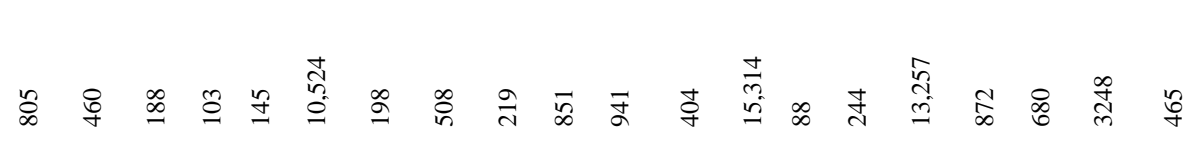

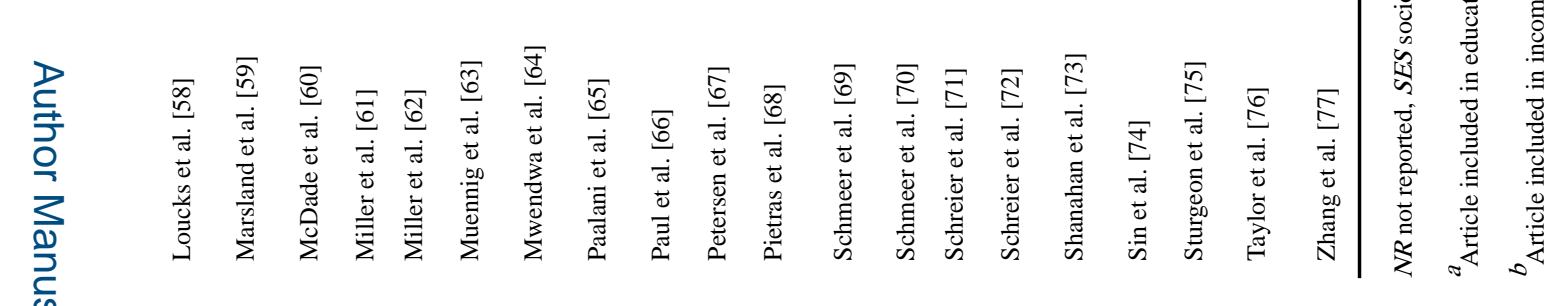
Mol Psychiatry. Author manuscript; available in PMC 2020 April 19. 